\title{
Análise da produção de estudos com métodos mistos na avaliação de serviços de saúde mental
}

Analysis of the production of mixed methods studies in the evaluation of mental health services

Análisis de la producción de estudios de métodos mixtos en la evaluación de servicios de salud mental

\section{Guilherme Emanuel Weiss Pinheiro', Angela Maria Pereira dos Santos ${ }^{\mathrm{II}}$, Luciane Prado KantorskiIII}

\begin{abstract}
Resumo: Objetivo: analisar os desenhos de pesquisa de métodos mistos produzidos sobre avaliação de serviços de saúde mental. Método: estudo de análise documental, realizado nos meses de abril a junho de 2019, o qual analisou os desenhos de pesquisas de métodos mistos de avaliação de serviços de saúde mental, realizadas entre 2006 e 2016, por um grupo de pesquisa em enfermagem. Resultados: são apresentados três estudos de métodos mistos de avaliação de serviços de saúde mental da região sul do Brasil, todos desenvolvem-se a partir de uma dinâmica de integração similar. São apresentadas as etapas de integração, os objetivos, a metodologia e os eixos analíticos. Considerações finais: a realização de estudos de métodos mistos possibilitou a identificação de pontos de integração e convergência entre as abordagens quantitativas e qualitativas. Ressalta-se o potencial da metodologia para estudos avaliativos.
\end{abstract}

Descritores: Pesquisa; Métodos; Avaliação em saúde; Enfermagem

Abstract: Objective: to analyze the mixed method research designs produced on mental health services evaluation. Method: a document analysis study, conducted from April to June 2019, which analyzed the research designs of mixed methods of evaluation of mental health services, carried out between 2006 and 2016, by a nursing research group. Results: three studies of mixed methods of evaluation of mental health services from the southern region of Brazil are presented, all developed from a similar integration dynamics. The integration steps, objectives, methodology and analytical axes are presented. Conclusions: conducting mixed method studies has enabled the identification of integration and convergence points between quantitative and qualitative approaches. The potential of the methodology for evaluative studies is emphasized.

Descriptors: Research; Methods; Health assessment; Nursing

Resumen: Objetivo: analizar los diseños de investigación de métodos mixtos producidos en la evaluación de servicios de salud mental. Método: un estudio de análisis de documentos, realizado de abril a junio de 2019, que analizó los

\footnotetext{
${ }^{\text {I }}$ Enfermeiro, Mestre em Ensino na Saúde pelo Programa de Pós-graduação em Ensino na Saúde da Universidade Federal de Ciências da Saúde de Porto Alegre, Professor EBTT no Colégio Politécnico da Universidade Federal de Santa Maria, Santa Maria, RS, Brasil, guilhermeewp@politecnico.ufsm.br, ORCID: http://orcid.org/0000-0003-0069-7023

${ }^{\text {II }}$ Psicóloga, graduada em psicologia pela Universidade Franciscana, Estudante do curso técnico em enfermagem do Colégio Politécnico da Universidade Federal de Santa Maria, Santa Maria, RS, Brasil, angelacooplantio@hotmail.com, ORCID: http://orcid.org/0000-0003-0181-608X

III Enfermeira, Doutora em Enfermagem pela Universidade de São Paulo, Professora na Universidade Federal de Pelotas, Pelotas, RS, Brasil, kantorski@uol.com.br, ORCID: http://orcid.org/0000-0001-9726-3162
} 
Análise da produção de estudos com métodos mistos na avaliação de serviços de saúde me... $\mid 2$

diseños de investigación de métodos mixtos de evaluación de servicios de salud mental, realizado entre 2006 y 2016, por un grupo de investigación en enfermería. Resultados: se presentan tres estudios de métodos mixtos de evaluación de servicios de salud mental de la región sur de Brasil, todos desarrollados a partir de una dinámica de integración similar. Se presentan los pasos de integración, objetivos, metodología y ejes analíticos. Consideraciones finales: la realización de estudios de métodos mixtos ha permitido identificar puntos de integración y convergencia entre enfoques cuantitativos y cualitativos. Se enfatiza el potencial de la metodología para estudios evaluativos.

Descriptores: Investigación; Métodos; Evaluación de salud; Enfermería

\section{Introdução}

Os métodos mistos de pesquisa são uma alternativa para os estudos na área das ciências sociais, humanas e em saúde. Neste contexto, as questões e os problemas de pesquisa têm chances de serem melhores respondidos e investigados. É considerado mais do que um método, sendo ampliado para uma metodologia que une as abordagens quantitativa e qualitativa em torno de um objeto de pesquisa. ${ }^{1}$ Para além disso, trata-se de uma metodologia que combina os métodos, as abordagens, a filosofia e a orientação do projeto de pesquisa. ${ }^{1}$ Como aspectos fundamentais na utilização dos métodos mistos estão: a coletânea análise de dados quantitativos e qualitativos; a combinação das duas formas de dados; a integração dos procedimentos no mesmo estudo; e a estruturação dos procedimentos a partir de visões de mundo filosóficas e teóricas. $^{2}$

A abordagem quantitativa produz dados como números e indicadores, sendo analisadas a partir da métrica e suas expressões. Sendo capazes de apresentar informações rápidas e confiáveis a respeito do objeto investigado. A abordagem qualitativa ao utilizar entrevistas abertas, observação, entre outras técnicas, têm o intuito de oferecer informações sobre a fala dos sujeitos entrevistados ou observados, proporcionando a visão de outras perspectivas sobre o objeto estudado e delineando aspectos subjetivos do fenômeno pesquisado. ${ }^{1}$

Chamada de abordagem multimétodo, a pesquisa de métodos mistos é definida como um conjunto de procedimentos de coleta, de análises e de combinação de técnicas quantitativas e qualitativas em torno de um mesmo desenho de pesquisa. A interação entre as abordagens é 
considerada um dos principais pressupostos, pois fornece melhores possibilidades de análise a partir disso. $^{2}$

As abordagens quantitativa e qualitativa mantêm um debate epistemológico e ontológico. Sendo assim, na visão positivista os métodos e técnicas das Ciências Sociais precisam se aproximar das Ciências Naturais, já o interpretativismo sustenta que as Ciências Sociais e Naturais se constituem como abordagens distintas e para isso necessitam de métodos e técnicas específicos. ${ }^{3}$ Contudo, há uma diferença entre as Ciências Naturais e as Ciências Sociais que é o significado, próprio desta última, sendo indispensável à interpretação da realidade social. ${ }^{3} \mathrm{Uma}$ vez que, as Ciências Sociais buscam se ocupar com a interpretação das ações dos sujeitos e revelam os significados subjetivos em sua análise. ${ }^{3}$

Os estudos com métodos mistos apresentam vantagens, entre as destacadas estão a integração entre as abordagens qualitativa e quantitativa e a possibilidade de retirar o melhor de cada uma para responder ao problema e as questões de pesquisa elencadas. ${ }^{3}$ Assim, a mescla das abordagens apresenta resultados positivos, pois cada uma colabora da sua forma para um melhor desenho de pesquisa, agregando resultados e compreensões complexas na investigação. ${ }^{3}$

Em estudo publicado em um periódico inglês, foram analisados os dez melhores recursos de pesquisa com métodos mistos em sistemas de saúde, constatando que se trata de uma terceira abordagem, cada vez mais popular e crescente nos sistemas de saúde. ${ }^{4}$ Demonstrando que, a abordagem quantitativa permite conhecer a extensão de determinada situação e também promovem o entendimento da representatividade dos resultados. $\mathrm{E}$ a qualitativa atua na perspectiva de ampliar a profundidade das compreensões, ofertando diferentes panoramas das partes interessadas e oferecem justificativas para os desempenhos dos sistemas de saúde. Com isso, é reforçado que os métodos mistos nas pesquisas na área da saúde são crescentes, uma vez que neste contexto é necessário abordar questões complexas, como fatores socioculturais, os quais 
Análise da produção de estudos com métodos mistos na avaliação de serviços de saúde me... 4

não poderiam ser suficientemente mensurados de forma isolada por uma abordagem específica, sendo necessária a integração das abordagens para compreender a complexidade dos fenômenos. ${ }^{4}$

Alguns pesquisadores constataram que há uma lacuna na produção de conhecimento em relação às pesquisas de métodos mistos, ${ }^{3,5-6}$ necessitando de mais empenho para o seu desenvolvimento. ${ }^{6}$ Neste sentido, defende-se que há a necessidade de integração e interação entre os componentes quantitativos e qualitativos de um estudo, sendo essencial para que algumas definições tomem corpo e se sustentem no contexto em que são investigadas. Isso é importante para expandir e fortalecer os resultados destes estudos, além de responder a questões de pesquisas e produzir conhecimentos de validade elevada. ${ }^{5}$

Um grupo de pesquisa realizou estudos de métodos mistos, no período de 2006 a 2016, na área da avaliação de serviços de saúde mental, com o intuito de realizar avaliações de ações, programas e serviços no interior das políticas públicas de saúde. Foram três projetos de pesquisas matriciais, multicêntricos e que avaliaram cerca de quarenta serviços de saúde da região sul do Brasil. Como questão de pesquisa este manuscrito apresenta a seguinte: quais as contribuições dos métodos mistos em pesquisa para avaliar serviços de saúde mental? Dessa forma, tem o objetivo de analisar os desenhos de pesquisa de métodos mistos produzidos sobre avaliação de serviços de saúde mental.

\section{Método}

Trata-se de um estudo de análise documental, realizado nos meses de abril a junho de 2019. A pesquisa documental é capaz de produzir novos conhecimentos, por meio da criação de formas diferenciadas de compreender os fenômenos, de conhecer como se relacionam e como vem sendo desenvolvidos, enquanto objetos de estudo. ${ }^{7}$ Também se acredita que para o desenvolvimento de estudos nas diversas áreas do conhecimento, a análise documental tenha sua relevância preservada, pois por meio dela os pesquisadores podem coletar, tratar e analisar 
as fontes de informações. ${ }^{8}$ Dessa forma, este estudo busca relacionar os desenhos das pesquisas que utilizaram métodos mistos para reconhecer objetos de estudos no campo da avaliação em saúde, em especial os serviços da rede de atenção psicossocial do sul do Brasil e suas contribuições para o campo da saúde e da enfermagem em saúde mental.

Os documentos analisados foram os relatórios de pesquisas de métodos mistos de avaliação de serviços de saúde mental, financiadas pelo Conselho Nacional de Desenvolvimento Científico e Tecnológico (CNPq), no período de 2006 a 2016. Os critérios de seleção foram: ter recebido financiamento de órgão de fomento via Ministério da Ciência e Tecnologia (MCT)CNPq/ MS-SCTIE-DECIT-CT-Saúde - Edital 07/2005 e ser pesquisa de método misto.

Estes critérios são justificados pelo fato de no ano de 2005 ter sido publicado o primeiro edital para financiamento de estudos avaliativos em saúde mental. A seleção ocorreu a partir das pesquisas selecionadas pelo edital supracitado, sendo escolhido um grupo, o único que realizou pesquisas de métodos mistos. O período de realização deste estudo está pautado em um recorte longitudinal de 10 anos, em que houve financiamento para pesquisa na área de avaliação de serviços de saúde mental.

Foi realizada a análise documental dos relatórios finais de três estudos que utilizaram métodos mistos de pesquisa, produzidos por um grupo de pesquisa em enfermagem, em saúde mental e em saúde coletiva de uma universidade pública, certificado pelo Diretório de Grupos de Pesquisa do Brasil do CNPq, ao longo de 10 anos. Os estudos analisados respeitaram as normativas éticas vigentes e foram aprovados por Comitês de Ética em Pesquisa.

\section{Resultados}

São apresentados os três estudos de avaliação de serviços de saúde mental da região sul do Brasil, os quais seguiram as características de desenho de método misto sequencial explicativo ou explanatório. O primeiro estudo, denominado como Estudo 1 nos quadros 1 e 2, foi realizado no período de 2006 a 2008, nos três Estados da região sul do Brasil, tendo como 
Análise da produção de estudos com métodos mistos na avaliação de serviços de saúde me... 6

objeto a avaliação dos Centros de Atenção Psicossocial (CAPS). O segundo estudo, denominado como Estudo 2, foi realizado no período de 2008 a 2010, no Rio Grande do Sul, tendo como objeto a avaliação das experiências inovadoras de composição de redes de atenção psicossocial. E o terceiro estudo, denominado Estudo 3, foi realizado no período de 2011 a 2016, nos três Estados da região sul do Brasil, sendo uma segunda edição do Estudo 1.

Os três estudos apresentaram uma dinâmica de integração similar. Os estudos de casos da etapa qualitativa foram selecionados a partir do compilado de dados quantitativos e que, conjuntamente, apresentaram melhor desempenho baseado nas normativas legais do Ministério da Saúde em relação aos serviços de atenção psicossocial. Além disso, foram observados os indicadores de estrutura, processo e resultado, comparados às legislações, assim, a decisão de escolha dos municípios ocorreu de forma coletiva e baseada nos dados quantitativos.

\begin{tabular}{|c|c|c|c|c|}
\hline \multirow[t]{2}{*}{ Pesquisa } & \multicolumn{2}{|r|}{ Quantitativo } & \multicolumn{2}{|r|}{ Qualitativo } \\
\hline & Objetivos & Metodologia & Objetivos & Metodologia \\
\hline $\begin{array}{l}\text { ESTUDO } 1 \\
(2006-2008)\end{array}$ & $\begin{array}{l}\text { Objetivo } \\
\text { Geral: } \\
\text { Avaliar a } \\
\text { estrutura, o } \\
\text { processo e o } \\
\text { resultado da } \\
\text { atenção em } \\
\text { saúde } \\
\text { desenvolvida } \\
\text { pelos CAPS } \\
\text { da Região Sul } \\
\text { do Brasil - } \\
\text { Rio Grande } \\
\text { do Sul, Santa } \\
\text { Catarina, } \\
\text { Paraná. }\end{array}$ & $\begin{array}{l}\text { Participantes: } \\
\text { - Usuários: } 1.162 \\
\text { - Familiares: } 936 \\
\text { - Trabalhadores: } 435 \\
\text { - Coordenadores: } 30 \\
\text { Delineamento: } 30 \text { CAPS } \\
\text { distribuídos entre os } \\
\text { Estados da região sul do } \\
\text { Brasil Estudo } \\
\text { Abordagem: estrutura, do } \\
\text { descritivo da estrua e } \\
\text { processo de trabalho e } \\
\text { organização da atenção em } \\
\text { saúde e estudo transversal } \\
\text { de avaliação dos resultados } \\
\text { da atenção em saúde } \\
\text { mental da região sul. } \\
\text { Análise: As análises foram } \\
\text { realizadas no software } \\
\text { (Software for Statistics and } \\
\text { Data Science) STATA, } \\
\text { incluindo análise estatística } \\
\text { univariada e bivariada. }\end{array}$ & $\begin{array}{c}\text { Objetivo } \\
\text { Geral: } \\
\text { Avaliar } \\
\text { qualitativamen } \\
\text { te Centros de } \\
\text { Atenção } \\
\text { Psicossocial da } \\
\text { Região Sul } \\
\text { (CAPS I, e II) } \\
\text { que estão } \\
\text { localizados nos } \\
\text { Estados do Rio } \\
\text { Grande do Sul, } \\
\text { Santa } \\
\text { Catarina, } \\
\text { Paraná. }\end{array}$ & $\begin{array}{l}\text { Participantes: } \\
\text { - Usuários: } 57 \\
\text { - Familiares: } 60 \\
\text { - Trabalhadores: } 88 \\
\text { Observação em diário de } \\
\text { campo: } 2005 \text { horas } \\
\text { Delineamento: } 5 \text { CAPS (3 } \\
\text { Rio Grande do Sul, } 1 \text { Santa } \\
\text { Catarina e } 1 \text { Paraná) } \\
\text { Abordagem: } \\
\text { Estudo de avaliação } \\
\text { qualitativa dos Centros de } \\
\text { Atenção Psicossocial da } \\
\text { Região Sul, através da } \\
\text { avaliação de quarta geração, } \\
\text { construtivista e responsiva, } \\
\text { e estudo de casos múltiplos. } \\
\text { Análise: } \\
\text { Comparativo Constante }\end{array}$ \\
\hline
\end{tabular}




\begin{tabular}{|c|c|c|c|c|}
\hline $\begin{array}{c}\text { ESTUDO } 2 \\
(2008-2010)\end{array}$ & $\begin{array}{c}\text { Objetivo } \\
\text { Geral: } \\
\text { Avaliar a } \\
\text { composição e } \\
\text { qualidade } \\
\text { das redes de } \\
\text { atenção } \\
\text { psicossocial } \\
\text { da Região Sul } \\
\text { do Brasil/RS. }\end{array}$ & $\begin{array}{l}\text { Participantes: } \\
\text { - Usuários: } 392 \\
\text { - Trabalhadores: } 209 \\
\text { - Coordenadores: } 14 \\
\text { Delineamento: } \\
\text { municípios do Estado do } \\
\text { Rio Grande do Sul } \\
\text { Abordagem: } \\
\text { descritivo para caracterizar } \\
\text { a estrutura e processo de } \\
\text { estruturação das redes de } \\
\text { atenção em saúde mental e } \\
\text { estudo transversal para } \\
\text { avaliar a autonomia dos } \\
\text { usuários das redes de } \\
\text { serviços em saúde mental } \\
\text { Análise: Por meio dos } \\
\text { softwares EPI-INFO e } \\
\text { STATA }\end{array}$ & $\begin{array}{c}\text { Objetivo } \\
\text { Geral: Avaliar } \\
\text { qualitativamen } \\
\text { te experiências } \\
\text { inovadoras de } \\
\text { composição de } \\
\text { redes de } \\
\text { atenção } \\
\text { psicossocial } \\
\text { que estão } \\
\text { localizados no } \\
\text { Estado do Rio } \\
\text { Grande do Sul. }\end{array}$ & $\begin{array}{l}\text { Participantes: } \\
\text { - Usuários: } 33 \\
\text { - Trabalhadores: } 21 \\
\text { - Gestores: } 23 \\
\text { Observação em diário de } \\
\text { campo: } 1400 \text { horas } \\
\text { Delineamento: } \\
\text { municípios do Estado do } \\
\text { Rio Grande do Sul } \\
\text { Abordagem: } \\
\text { Estudo de avaliação } \\
\text { qualitativa de experiências } \\
\text { inovadoras de composição } \\
\text { de redes de atenção } \\
\text { psicossocial - através da } \\
\text { avaliação de quarta geração, } \\
\text { construtivista e responsiva, } \\
\text { metodologia de análise de } \\
\text { redes do cotidiano e estudo } \\
\text { de casos múltiplos. } \\
\text { Análise: } \\
\text { Comparativo Constante }\end{array}$ \\
\hline $\begin{array}{l}\text { ESTUDO } 3 \\
(2011-2016)\end{array}$ & $\begin{array}{c}\text { Objetivo } \\
\text { Geral: } \\
\text { Avaliar a } \\
\text { estrutura, o } \\
\text { processo e o } \\
\text { resultado da } \\
\text { atenção em } \\
\text { saúde } \\
\text { desenvolvida } \\
\text { pelos CAPS } \\
\text { da região sul } \\
\text { do Brasil - } \\
\text { RS, SC, PR. }\end{array}$ & $\begin{array}{l}\text { Participantes: } \\
\text { - Usuários: } 1597 \\
\text { - Familiares: } 1262 \\
\text { - Trabalhadores: } 546 \\
\text { Delineamento: } \\
\text { Todos os } 308 \text { CAPS (I, II e } \\
\text { III), CAPSI e CAPS Álcool } \\
\text { e Drogas (AD) da região sul. } \\
\text { Abordagem: Estudo } \\
\text { descritivo para identificar } \\
\text { as características da } \\
\text { estrutura, processo de } \\
\text { atendimento e estudo } \\
\text { transversal para avaliar os } \\
\text { resultados da atenção em } \\
\text { saúde mental da região sul. } \\
\text { Análise: As análises foram } \\
\text { realizadas nos softwares } \\
\text { EPI-INFO e STATA, } \\
\text { incluindo análise } \\
\text { univariada e bivariada }\end{array}$ & $\begin{array}{c}\text { Objetivo } \\
\text { Geral: Avaliar } \\
\text { qualitativamen } \\
\text { te Centros de } \\
\text { Atenção } \\
\text { Psicossocial da } \\
\text { Região Sul do } \\
\text { Brasil. }\end{array}$ & $\begin{array}{l}\text { Participantes: } \\
\text { - Usuários: } 32 \\
\text { - Familiares: } 34 \\
\text { - Trabalhadores: } 45 \\
\text { Observação em diário de } \\
\text { campo: } 900 \text { horas } \\
\text { Delineamento: } 3 \text { CAPS (1 } \\
\text { Rio Grande do Sul, } 1 \text { Santa } \\
\text { Catarina e } 1 \text { Paraná) } \\
\text { Abordagem: } \\
\text { Estudo de avaliação } \\
\text { qualitativa dos Centros de } \\
\text { Atenção Psicossocial da } \\
\text { Região Sul, através da } \\
\text { avaliação de quarta geração, } \\
\text { construtivista e responsiva, } \\
\text { e estudos de casos } \\
\text { múltiplos Método } \\
\text { Análise: } \\
\text { Comparativo Constante }\end{array}$ \\
\hline
\end{tabular}

Quadro 1: Comparação dos objetivos e a metodologia de coleta de dados dos estudos de métodos mistos, 2019. 
Análise da produção de estudos com métodos mistos na avaliação de serviços de saúde me... $\mid 8$

Os estudos trabalharam com marcadores de avaliação e a partir disso ocorreu a integração entre os marcadores quantitativos e qualitativos. Mesmo que estes marcadores foram gerados com base em instrumentos específicos de cada abordagem, a integração dos dados culminou com a discussão nos resultados. Também é importante ressaltar que os estudos foram construídos a partir da triangulação de dados com entrevistas, análises de documentos, grupos e observação. Com base nisso, a integração ocorreu, da mesma forma, quando a etapa qualitativa ofereceu elementos para o aprofundamento e entendimento de lacunas elencadas na etapa quantitativa.

\begin{tabular}{|c|c|c|}
\hline & Marcadores Quantitativos & Marcadores Qualitativos \\
\hline $\begin{array}{l}\text { ESTUDO } 1 \\
(2006-2008)\end{array}$ & $\begin{array}{l}\text { Estrutura: estrutura física, recursos materiais, } \\
\text { recursos humanos, recurso técnico, sistema de } \\
\text { referência, alimentação, medicamentos. } \\
\text { Processo: adequação da atenção, território, } \\
\text { diversificar atividades, frequência das } \\
\text { atividades, assembleias. } \\
\text { Resultado: satisfação (trabalhadores, familiares } \\
\text { e usuários), características individuais. }\end{array}$ & $\begin{array}{l}\text { Estrutura: ambiência e recursos humanos. } \\
\text { Processo de trabalho: atividades como } \\
\text { suporte terapêutico, equipe, características } \\
\text { e organização do trabalho, plasticidade do } \\
\text { serviço, inserção da família. } \\
\text { Resultado: resultado da atenção } \\
\text { psicossocial. } \\
\text { Políticas públicas, gestão e articulação da } \\
\text { rede de saúde, relação da sociedade com o } \\
\text { fenômeno da loucura. }\end{array}$ \\
\hline $\begin{array}{c}\text { ESTUDO } 2 \\
(2008-2010)\end{array}$ & $\begin{array}{l}\text { Análise da sociabilidade dos usuários, do } \\
\text { suporte social, do inventário de habilidades de } \\
\text { vida independente. } \\
\text { Caracterização dos serviços quanto ao início } \\
\text { das atividades, ao horário, à estrutura, à } \\
\text { articulação com território, aos dispositivos de } \\
\text { garantia de cidadania, ao atendimento ao } \\
\text { usuário, à supervisão, à referência e contra } \\
\text { referência, à distribuição de medicação e } \\
\text { fornecimento de receitas, ao atendimento de } \\
\text { crise, ao atendimento a família, à reunião de } \\
\text { equipe e à articulação com a rede. }\end{array}$ & $\begin{array}{l}\text { Caracterização dos municípios, da rede de } \\
\text { atenção à saúde e dos serviços de saúde } \\
\text { mental. } \\
\text { Estudo da Rede de Atenção à Saúde, a } \\
\text { estrutura, fluxo e articulação. } \\
\text { Mapeamento da rede social dos usuários. } \\
\text { Fortalecimento da rede social dos usuários. } \\
\text { Maleabilidade e flexibilidade da rede. } \\
\text { Rede quente e rede social. } \\
\text { Marcadores de Inovação da Rede de Saúde } \\
\text { Mental }\end{array}$ \\
\hline $\begin{array}{c}\text { ESTUDO } 3 \\
(2011-2016)\end{array}$ & $\begin{array}{l}\text { Estrutura: estrutura física, recursos materiais, } \\
\text { recursos humanos, recurso técnico, sistema de } \\
\text { referência, alimentação, medicamentos. } \\
\text { Processo: adequação da atenção, território, } \\
\text { diversificar atividades, frequência das } \\
\text { atividades, assembleias. } \\
\text { Resultado: satisfação (trabalhadores, familiares } \\
\text { e usuários), características individuais. }\end{array}$ & $\begin{array}{l}\text { Estrutura: ambiência e recursos humanos. } \\
\text { Processo de Trabalho e a rede de atenção } \\
\text { psicossocial no CAPS: atividades como } \\
\text { suporte terapêutico, equipe, características } \\
\text { e organização do trabalho, plasticidade do } \\
\text { serviço, inserção da família. } \\
\text { Resultado: resultado da atenção } \\
\text { psicossocial. }\end{array}$ \\
\hline
\end{tabular}

Quadro 2: Comparação dos marcadores dos estudos de métodos mistos, 2019. 


\section{Discussão}

Os estudos analisados apresentam um diferencial na construção de seus desenhos, pois são pesquisas de métodos mistos. Embora cada abordagem apresente técnicas e métodos diferentes, além de base teóricas independentes na construção de seus desenhos, o método misto busca à integração em diferentes momentos. A abordagem quantitativa, nestes casos, tem base na epidemiologia e no positivismo e a qualitativa está baseada na avaliação de quarta geração, construtivista e responsiva e nos estudos de casos múltiplos. Entretanto, as abordagens convergiram para um processo integrativo, desde a eleição do cenário da etapa qualitativa como na discussão dos resultados.

As características do positivismo concentram-se em torno da realidade singular (ontologia), da distância e da imparcialidade (epistemologia), dos testes de teorias a priori (metodologia) e das definições acordadas nas variáveis para escrita dos relatórios (retórica). ${ }^{2}$ Objetiva, além disso, ao teste de teorias ou a descrição de experiências, por meio da observação ou da medição, para controlar e predizer as forças que cercam o objeto estudado. Tem uma visão dura da realidade pura, passível de ser capturada e prevista, assim o positivismo utiliza a dedução para confirmar teorias, utilizando métodos de coleta e análise de dados quantitativos. ${ }^{9}$

O construtivismo, por sua vez, concentra-se em realidades múltiplas (ontologia), na proximidade com o objeto de estudo (epistemologia), sendo indutivo, pois parte das visões dos sujeitos e depois chega aos padrões (metodologia) e constrói seus relatórios a partir de um estilo literário informal (retórica). Está associado à abordagem qualitativa e funciona com uma visão de mundo diferente. Uma vez que os participantes apresentam seus entendimentos, a pesquisa se constrói baseada nas perspectivas individuais para os entendimentos amplos. ${ }^{2}$ A partir da subjetividade e da realidade vivenciada pelos sujeitos da pesquisa. 
Análise da produção de estudos com métodos mistos na avaliação de serviços de saúde me... | 10

Os métodos mistos consistem na coleta, análise e integração dos dados qualitativos e quantitativos, o que contribui para a melhor compreensão do problema de pesquisa. Não se trata apenas da obtenção de dados, mas de um processo de integração entre eles. ${ }^{2,5,10}$

Estudiosos na área de metodologia vêm ressaltando que a integração entre as abordagens quantitativa e qualitativa é peça central nos estudos de métodos mistos, sendo um processo intencional. Há uma independência das abordagens em relação às questões e as hipóteses de pesquisa que ao mesmo tempo são comuns. Os pesquisadores percebem os benefícios dos métodos mistos, ao observar que a integração produz um todo maior do que a soma das contribuições individuais das abordagens qualitativa e quantitativa. ${ }^{6}$

Para exemplificar a forma de como a integração é possível e para facilitar didaticamente, autores trabalharam na sistematização de desenhos básicos de projetos, podendo ser: sequenciais exploratórios, sequenciais explicativos ou explanatórios e convergentes.4.-6,10-11 Ainda, pode-se acrescentar os projetos incorporados ou aninhados, ${ }^{4-5,11} \mathrm{o}$ transformador e o multifase..$^{4-5}$

O sequencial exploratório inicia com a fase qualitativa de coleta e de análise dos dados que irá basear a próxima fase quantitativa, podendo gerar generalizações. O sequencial explicativo ou explanatório parte de uma fase quantitativa de coleta e de análise dos dados, o qual terá prosseguimento com a etapa qualitativa, buscando explicar os dados quantitativos. E o convergente compreende as coletas e análises quantitativas e qualitativas em momentos próximos e prossegue com a análise integrada, os dados têm constituições diferentes, mas complementares. Além disso, reforçam que a integração das abordagens quantitativa e qualitativa pode ocorrer de outras formas: discussão dos resultados quantitativos com uma abordagem qualitativa; construção de instrumentos quantitativos, a partir de resultados qualitativos; fusão dos resultados; ou incorporação de uma abordagem dentro da outra. .-6,10-11 $^{-1}$

Quando se fala em integração das abordagens para a construção de projetos de métodos mistos, podem-se considerar outros desenhos, chamados desenhos avançados, a saber: múltiplos 
estágios; estudos de intervenção; estudo de caso; pesquisa transformadora e pesquisa participativa; estudos de avaliação; ou desenvolvimento de aplicativo/website/plataforma. ${ }^{10}$

Assim, os estudos analisados utilizaram uma abordagem com métodos mistos, seguindo o desenho básico sequencial explicativo ou explanatório, que tem como objetivo utilizar a abordagem qualitativa para ajudar a explicar os resultados da abordagem quantitativa. Deste modo, os estudos tiveram suas etapas quantitativas realizadas em um primeiro momento e após isso, os resultados foram base para a escolha dos serviços que receberiam a etapa qualitativa, sendo destacados aqueles com as experiências positivas de atenção à saúde mental, a partir dos dados quantitativos e da análise das normativas ministeriais. Os dados qualitativos, por sua vez ajudaram a explicar os resultados quantitativos e ofereceram estudo em profundidade dos serviços destacados na primeira etapa. Ao final ocorreu a integração dos dados na apresentação dos relatórios de pesquisa.

É preciso prosseguir discutindo os dados apresentados no quadro 1, iniciando pelos objetivos dos estudos, há uma combinação do processo avaliativo, no estudo quantitativo a avaliação se deu por meio das condições da estrutura, do processo e do resultado da atenção psicossocial e com a utilização de alguns termos como: fatores, causas, medidas, determinantes, correlações, tendências, níveis e magnitudes. E no qualitativo a avaliação culmina com os significados, as experiências e os olhares dos atores envolvidos na atenção psicossocial. Assim, a pesquisa de métodos mistos precisa combinar as abordagens quantitativa e qualitativa, possibilitando a compreensão das experiências dos sujeitos e as relações entre as variáveis. ${ }^{10}$

Ao analisar os objetivos das abordagens dos estudos, são convergentes no sentido de explorar aspectos comuns nos eixos de análise: estrutura, processo e resultado do trabalho desenvolvido nos serviços, mas também, abordam aspectos diferentes no interior de cada eixo de análise como: infraestrutura física, recursos materiais, recursos humanos, recursos técnicos, sistema de referência, alimentação e medicamentos (estrutura); adequação da atenção, território, 
Análise da produção de estudos com métodos mistos na avaliação de serviços de saúde me... | 12

diversificação e frequência das atividades e assembleias (processo); e satisfação e características individuais (resultado). Os objetivos dos estudos quantitativos partem de conceitos que possuem variáveis como estrutura, processo e resultado, que são variáveis objetivas definidas. Já os qualitativos surgem a partir de um contexto mais amplo como a avaliação da qualidade do serviço, o que é algo subjetivo e que cada entrevistado irá pautar seu lugar de fala e avaliar a partir de suas experiências de ambiência, plasticidade do serviço, inserção da família, organização do trabalho, resultado da atenção psicossocial, entre outras.

$\mathrm{Na}$ abordagem quantitativa, as variáveis precisam ter relações estabelecidas e a partir disso, sendo provadas ou refutadas como hipóteses iniciais. Enquanto isso, nas qualitativas as ideias e definições básicas precisam ser identificadas, baseando o estudo e os principais conceitos de sensibilização sobre os quais os dados serão construídos. ${ }^{12}$

Outro aspecto possível de destacar, no quadro 1, são as convergências entre os sujeitos do estudo (trabalhadores de saúde, usuários dos serviços de saúde mental e familiares) e de unidade de análise (os CAPS, em ambas as etapas). Nos estudos analisados, a etapa quantitativa foi feita com o mesmo grupo de sujeitos numa amostra aleatória, o que garantiu a equiprobabilidade, pois todos os serviços tiveram a mesma chance de serem selecionados em na etapa posterior. ${ }^{3}$

No final desta etapa, foram selecionados serviços (unidades de análise) e entre os sujeitos que participaram da primeira etapa foram selecionados intencionalmente, dentre estes sujeitos, a partir de critérios pré-estabelecidos, aqueles sujeitos que participariam da etapa qualitativa. No que diz respeito à unidade de análise é necessário definir qual será a unidade básica da pesquisa, após, é preciso garantir que a mesma seja homogênea. ${ }^{3}$ Ressalta-se a integração na pesquisa de métodos mistos, uma vez que o processo de amostragem quantitativo e qualitativo garante que os sujeitos do estudo e a unidade de análise sejam os mesmos. ${ }^{10}$

Destacam-se as diferenças de amostragem entre os estudos no que diz respeito ao número de serviços ou municípios e de sujeitos que participaram das etapas das pesquisas. No 
estudo 1, na etapa quantitativa foram 30 CAPS e 2.563 pessoas entrevistadas, na qualitativa foram cinco estudos de caso em cinco CAPS e 205 pessoas entrevistadas. No estudo 2, na quantitativa foram cinco municípios e 615 pessoas entrevistadas e na qualitativa, dois municípios e 77 pessoas entrevistadas. E no estudo 3, na quantitativa foram 308 CAPS e 3.405 pessoas entrevistadas e na qualitativa, três CAPS e 111 pessoas entrevistadas.

Assim, a abordagem qualitativa parte da observação próxima, sendo uma forma de estudo que precisa do aprofundamento e de detalhes para construir suas bases de investigação, orquestrada por estudos intensivos e com número reduzido de casos. Já a quantitativa tem a prerrogativa de trabalhar com amostras maiores, ou seja, mais numerosa para que sua representatividade tenha validade em seu método. ${ }^{13}$

Na mesma direção, o exame da associação entre as variáveis pode gerar generalizações para determinada população pela utilização de inferências estatísticas. Desta forma, a abordagem quantitativa foca sua análise em amostras maiores, mas não apresentam a compreensão da individualidade. Ao passo que a abordagem qualitativa é dotada de descrições detalhadas dos fenômenos estudados, apresentando o contexto e podendo focar sua análise no aprofundamento da individualidade, porém seus resultados não são generalizáveis. ${ }^{14}$

Em relação aos instrumentos e às técnicas utilizadas nas diferentes etapas dos estudos é possível observar que na abordagem quantitativa os instrumentos foram questionários com escalas e outras questões fechadas para avaliar, entre outros aspectos, a estrutura, o processo e o resultado dos serviços de saúde. À medida que na abordagem qualitativa foram utilizados outros instrumentos como a entrevista semiestruturada, com questões abertas, a observação participante, o grupo de validação. Isso possibilitou conhecer e compreender as particularidades culturais, econômicas, organizacionais, políticas e sociais dos serviços estudados a partir da abordagem qualitativa, e integrando isto a abordagem qualitativa, a qual teve a capacidade de mensurar as associações entre os fatores que compunham a avaliação dos diferentes aspectos abordados. ${ }^{14}$ 
Análise da produção de estudos com métodos mistos na avaliação de serviços de saúde me... | 14

A utilização de diferentes técnicas ou instrumentos não é escolhida ao acaso ou aleatoriamente, mas sim baseada no referencial teórico que cada abordagem carrega intrinsecamente. Nesse sentido, há discussões no que diz respeito às bases utilizadas em cada abordagem, entretanto é necessário ter ciência de que ao estudar a realidade social, a subjetividade que permeia este meio precisa ser valorizada, e no momento de trabalhar com indicadores quantitativos e com variáveis, a visão positivista se faz importante e necessária para que os resultados sejam plausíveis ao objeto que está sendo investigado. ${ }^{3}$

O que vai caracterizar as pesquisas analisadas que contam com uma etapa qualitativa e outra quantitativa, em pesquisas com métodos mistos são justamente a capacidade de integração no interior da pesquisa que perpassa desde os objetivos e os métodos até a complementaridade dos resultados. Alguns teóricos apontam algumas características básicas para que a integração seja garantida, a saber: a corroboração ou convergência (os resultados de uma abordagem colaboram com os de outra); a complementaridade (uma abordagem auxilia na melhoria/esclarecimento dos resultados de outra); o desenvolvimento (resultados de uma abordagem informa os resultados da outra - projetar um instrumento, delimitar uma amostragem); a iniciação (ajuda na descoberta de paradoxos e contradições da outra); a expansão (profundidade e amplitude são expandidas); e a discordância (quando há conflito entre as abordagens). ${ }^{10-11}$

A integração e a convergência são características dos estudos multimétodos e quanto mais convergentes e integrados forem os resultados mais consistente será a pesquisa. Assim, a integração tem como intuito, dentre outros, o de potencializar as informações incorporadas em uma pesquisa, beneficiando e aprimorando os achados do estudo. ${ }^{3}$

No quadro 2, é possível notar as aproximações, as diferenças e as complementaridades entre os marcadores utilizados na fase de análise, ao observar a avaliação de estrutura, por exemplo, na abordagem quantitativa, nota-se a questão da infraestrutura física - por exemplo número de salas e dimensão do espaço; dos recursos materiais, dos recursos humanos - por 
exemplo, número de profissionais, carga horária dos profissionais, das características do serviço, entre outros. Com isso, é possível verificar se a estrutura está adequada ou não e se apresenta o mínimo exigido pelas normativas e legislações dos serviços de saúde mental vigentes.

Já na abordagem qualitativa observa-se a ambiência, no que diz respeito à estrutura, com detalhes subjetivos dos sujeitos envolvidos nestes processos avaliativos, ou seja, estão expressos como os atores veem a estrutura, como se sentem nela, se tem privacidade, como se sentem acolhidos, quais as suas percepções e preocupações. Assim, o quantitativo atua sobre as variáveis objetivas no processo avaliativo e o qualitativo sobre as questões subjetivas e intersubjetivas da avaliação. ${ }^{12}$ Os exemplos destes estudos avaliativos contribuem para demonstrar a capacidade de complementação, de integração e de fortalecimento dos resultados apresentados pelas diferentes abordagens ao se constituir em um estudo de métodos mistos.

A partir desta constatação é possível notar a ligação entre as abordagens, ou seja, como o quantitativo contribui para o qualitativo e vice-versa. A questão principal nesta ligação é como ocorre esta integração entre as abordagens para que o método misto se consolide como uma alternativa a pesquisa. ${ }^{3,10,15}$

No mesmo sentido, o lugar que o pesquisador ocupa em cada uma das abordagens precisa ser levado em conta, em especial, ao discutir os resultados de investigações na área da avaliação dos serviços de saúde. $\mathrm{Na}$ abordagem qualitativa o pesquisador participa do processo ativamente, seus valores se incorporam como parte da pesquisa e na quantitativa o pesquisador e seus valores mantém-se afastados do objeto avaliado. ${ }^{12}$ Essa descoberta permite que os dados também sejam tratados de formas diferenciadas em cada abordagem, uma vez que são obtidos de forma distinta. Assim, reforça a capacidade colaborativa de uma abordagem com a outra e vice-versa.

$\mathrm{Na}$ experiência destes estudos, é possível notar que as abordagens se subsidiam e se complementam. Os estudos quantitativos, realizados primeiramente, apresentaram o panorama geral dos serviços de saúde mental e permitiram generalizações no âmbito regional, oferecendo, 
Análise da produção de estudos com métodos mistos na avaliação de serviços de saúde me... | 16

posteriormente, recursos para que o qualitativo fosse realizado. A partir disso, se tomaram por base os estudos quantitativos e foram escolhidos, a partir de critérios objetivos, os melhores resultados em relação aos marcadores e as experiências de atenção à saúde mental, para que fossem realizados os estudos de casos e a avaliação de quarta geração, com abordagem qualitativa.

A partir desta experiência, de um estudo quantitativo subsidiar um estudo qualitativo na área da avaliação dos serviços de saúde, é importante ressaltar que os sistemas de saúde vêm tornando-se algo complexo devido a diversos fatores (aumento da população, envelhecimento, adoecimento, restrições orçamentárias, entre outros). Com isso, os problemas de pesquisa acompanham a complexidade das questões dos sistemas de saúde, desafiando os pesquisadores a buscar formas de pesquisas mais abrangentes. ${ }^{11}$ Assim, as pesquisas de métodos mistos, que são multidimensionais e mais abrangentes do que as abordagens convencionais, contribuem para buscar respostas às necessidades dos sistemas de saúde. Assim, a pesquisa de métodos mistos permite aos pesquisadores explorar os fenômenos complexos com detalhes, ${ }^{11}$ respeitando os princípios de cada abordagem e agregando conforme a necessidade requerida para a investigação do objeto de pesquisa.

Nesse sentido, a enfermagem possui destaque na realização destes estudos, uma vez que os estudos analisados, neste artigo, foram coordenados por enfermeiras e é possível constatar que a enfermagem concentra funções de gestão no interior dos serviços de saúde. Corroborando com isto, um estudo multicêntrico aponta que a equipe de enfermagem é a categoria profissional que mais exerce funções centrais na rede de atenção à saúde e, na Estratégia Saúde da Família (ESF), estes profissionais estão presentes em todas as equipes, impactando a qualidade da assistência prestada. ${ }^{16}$

Endossando esta afirmação, resgata-se estudo que verificou a contribuição da utilização de indicadores de saúde no cotidiano do trabalho de enfermeiros gerentes da ESF para o planejamento e para a avaliação em saúde. ${ }^{17}$ Assim, a integração dos indicadores qualitativos e 
quantitativos são importantes, inclusive no fazer em saúde, avançando para além da sua aplicabilidade no campo da pesquisa, ofertando a compreensão de métodos mistos para avaliar os indicadores de saúde.

\section{Considerações finais}

A utilização de métodos mistos em pesquisas é algo inovador e vem ganhando espaço na área da saúde. É importante destacar que a integração e a colaboração no interior dos estudos com métodos mistos o validam como metodologia da pesquisa potente e necessária para a investigação em saúde.

Com este artigo foi possível analisar os estudos de métodos mistos realizados por um grupo de pesquisa, apresentando os pontos de integração e convergência entre as abordagens e discutindo como cada uma favorece o processo avaliativo. Com isso, foi possível demonstrar as principais contribuições da integração das abordagens quantitativa e qualitativa, na investigação de um objeto de pesquisa, no caso a avaliação de serviços de saúde mental.

Este estudo apresenta limitações no que diz respeito à análise da realidade de um grupo de pesquisa específico, em um campo específico da avaliação de serviços. Entretanto, é interessante potencializar estudos nesta área para que os métodos mistos sejam reconhecidos como metodologia viável e promissora na área da saúde.

Para além da integração e complementação das abordagens, foi possível verificar a postura mista e colaborativa que esta metodologia oferece, oportunizando um olhar complexo e abrangente do objeto a ser estudado, fortalecendo os resultados encontrados. Além do mais, estas pesquisas apresentaram contribuições, com a utilização dos métodos mistos, para a área da saúde, pois a avaliação dos serviços contribui para a qualidade da assistência prestada, para a consolidação da rede de atenção à saúde e para o cuidado em enfermagem. 
Análise da produção de estudos com métodos mistos na avaliação de serviços de saúde me... | 18

\section{Referências}

1. Creswell JW. Investigação qualitativa e projeto de pesquisa: escolhendo entre cinco abordagens. Porto Alegre (RS): Penso; 2014.

2. Creswell JW, Clark VLP. Pesquisa de métodos mistos. Porto Alegre (RS): Penso; 2013.

3. Paranhos R, Figueiredo Filho DB, Rocha EC, Silva Júnior JA, Freitas D. Uma introdução aos métodos mistos. Sociologias [Internet]. 2016 maio-ago [acesso em 2019 jun 20];42(18):384-411. Disponível em: http://www.scielo.br/scielo.php?pid=S1517-45222016000200384\&script=sci_abstract\&tlng=pt doi:10.1590/15174522-018004221

4. Ozawa S, Pongpirul K. 10 best resources on... mixed methods research in health systems. Health Policy Plan [Internet]. 2013 abr [acesso em 2019 jun 18];29(3):323-7. Disponível em: https://www.ncbi.nlm.nih.gov/pubmed/23564372 doi:10.1093/heapol/czt019

5. Schoonenboom J, Johnson RB. How to construct a mixed methods research design. Kolner Z Soz Sozpsychol [Internet]. 2017 [acesso em 2019 jun 18];69:107-31. Disponível em: https://link.springer.com/article/10.1007/s11577-017-0454-1 doi:10.1007/s11577-017-0454-1

6. Guetterman TC, Fetters MD, Creswel JW. Integrating quantitative and qualitative results in health science mixed methods research through joint displays. Ann Fam Med [Internet] 2015 nov-dez [acesso em 2019 jun 20];13(6):554-61. Disponível em: https://www.ncbi.nlm.nih.gov/pubmed/26553895 doi:10.1370/afm.1865

7. Sá-Silva JR, Almeida CD, Guindani JF. Pesquisa documental: pistas teóricas e metodológicas. Rev Bras Hist Ciênc Soc [Internet]. 2009 jan-jun [acesso em 2019 maio 11];1(1):1-15. Disponível em: https://www.rbhcs.com/rbhcs/article/view/6/pdf

8. Garcia Junior EF, Medeiros S, Augusta C. Análise documental: uma metodologia da pesquisa para a Ciência da Informação. Temática [Internet]. 2017 jul [acesso em 2019 maio 11];13(7):138-50. Disponível em: http://www.periodicos.ufpb.br/ojs2/index.php/tematica/article/view/35383/18042

9. Silva HCC, Siqueira AO, Araújo MAV, Dornelas JS. Sejamos pragmáticos: pesquisas em sistemas de informação com relevância e rigor. iSys Rev Bras Sist Inf [Internet]. 2017 dez [acesso em 2019 jun 24];10(4):66-79. Disponível em: http://www.seer.unirio.br/index.php/isys/article/view/6664/6252

10. Dal-Farra RA, Fetters MD. Recentes avanços nas pesquisas com métodos mistos: aplicações nas áreas de Educação e Ensino. Acta Sci [Internet]. 2017 maio-jun [acesso em 2019 jun 15];19(3):466-92. Disponível em: https://www.researchgate.net/publication/325090710_Recentes_avancos_nas_pesquisas_com_metodos_mistos _aplicacoes_nas_areas_de_Educacao_e_Ensino_Recent_advances_in_mixed_methods_research_Applications _in_the_fields_of_Education_and_Teaching 
11. Halcomb E, Hickman L. Mixed methods research. Nurs Stand [Internet]. 2015 [acesso em 2019 maio 20];29(32):41-7. Disponível em: https://ro.uow.edu.au/cgi/viewcontent.cgi?article=3676\&context=smhpapers doi:10.7748/ns.29.32.41.e8858

12. Sautu R. Todo es teoría: objetivos y métodos de investigación. Buenos Aires: Lumiere; 2005.

13. Cardano M. Métodos qualitativos para pesquisa em saúde. J Nurs Health [Internet]. 2017 set-dez [acesso em 2019 jun 18];7(3):e177306. Disponível em: https://periodicos.ufpel.edu.br/ojs2/index.php/enfermagem/article/view/12847/8061

14. Galvão MCB, Pluye P, Ricarte ILM. Métodos de pesquisa mistos e revisões de literatura mistas: conceitos, construção e critérios de avaliação. InCID Rev Ciênc Inf Doc [Internet]. 2017 set-fev [acesso em 2019 jun 2018];8(2):4-24. Disponível em: http://www.revistas.usp.br/incid/article/view/121879/133611 doi: 10.11606/issn.2178-2075.v8i2p4-24

15. Almalk S. Integrating quantitative and qualitative data in mixed methods research: challenges and benefits. J Educ Learn [Internet]. 2016 jul [acesso em 2019 jun 2018];5(3):288-96. Disponível em: https:/files.eric.ed.gov/fulltext/EJ1110464.pdf doi:10.5539/jel.v5n3p288

16. Pires DEP, Machado RR, Soratto J, Schere MA, Gonçalves ASR, Trindade LL. Cargas de trabalho da enfermagem na saúde da família: implicações no acesso universal. Rev Latinoam Enferm [Internet]. 2016 mar [acesso em 2019 jun 21];24:e2677. Disponível em: http://www.scielo.br/pdf/rlae/v24/pt_0104-1169-rlae0992-2682.pdf doi:10.1590/1518-8345.0992.2682

17. Paes LG, Bellato TMS, Machado BP, Lima SBS. O uso de indicadores como ferramenta de gestão na estratégia saúde da família. Rev Enferm UFSM [Internet]. 2015 jan-mar [acesso em 2019 jun 21];5(1):40-9. Disponível em: https://periodicos.ufsm.br/reufsm/article/view/14150/pdf doi:10.5902/2179769214150

\section{Autor correspondente}

Guilherme Emanuel Weiss Pinheiro

E-mail: guilhermeewp@politecnico.ufsm.br

Endereço: Avenida Roraima, $n^{\circ}$ 1000, Campus UFSM, Prédio 70 G, sala G321A, Bairro Camobi, Santa Maria - RS CEP: $97105-900$

\section{Contribuições de Autoria}

1 - Guilherme Emanuel Weiss Pinheiro

Concepção e planejamento da pesquisa, análise e interpretação dos dados, redação e revisão crítica.

Autor 2 - Angela Maria Pereira dos Santos

Análise e interpretação dos dados e revisão crítica. 
Análise da produção de estudos com métodos mistos na avaliação de serviços de saúde me... | 20

3 - Luciane Prado Kantorski

Concepção e planejamento da pesquisa, análise e interpretação dos dados, redação e revisão crítica.

\section{Como citar este artigo}

Pinheiro GEW, Santos AMP, Kantorski LP. Análise da produção de estudos com métodos mistos na avaliação de serviços de saúde mental. Rev. Enferm. UFSM. 2019 [Acesso em: Anos Mês Dia];vol.9 (Esp) e3: 1-20. DOI:https://doi.org/10.5902/2179769238707 